\title{
SIGNIFICADO DE LA SEXUALIDAD EN ADULTOS JOVENES SOMETIDOS A QUIMIOTERAPIA POR NEOPLASIA TESTICULAR
}

\author{
MEANING OF SEXUALITY IN YOUNG ADULTS UNDERGOING \\ CHEMOTHERAPY FOR TESTICULAR NEOPLASM
}

\author{
Carmen Luz Muñoz Zambrano* \\ Ximena Osorio Spuler ${ }^{* *}$
}

\begin{abstract}
RESUMEN
El cáncer es una enfermedad que provoca un fenómeno social y humano muy importante; así, el cáncer testicular es el tumor maligno más común en hombres entre los 15-35 años, siendo una de las neoplasias sólidas más curables. La presente investigación cualitativa descriptiva, con enfoque fenomenológico, pretende describir el significado que otorgan a su sexualidad seis adultos jóvenes que recibieron tratamiento con quimioterapia por cáncer testicular en la ciudad de Puerto Montt, de marzo a diciembre del 2010, respecto de su autoestima, relación de pareja y vida sexual. El análisis de datos se realizó utilizando el programa computacional Atlas Ti. Se observó que el significado que le otorga el adulto joven a su sexualidad está comprendido por la valoración propia y de su entorno, en función de la red de apoyo que cada uno tiene, donde la vida sexual sigue siendo importante, pero no trascendente ni primordial. La pareja es un aspecto que contribuye a la formación de un proyecto de vida y es en esa relación de pareja que la vida sexual se torna relevante. Por lo anterior, los efectos físicos ocasionados por el tratamiento, a pesar de las inevitables implicancias que conlleva, no condicionan la autoimagen, vida sexual ni la relación de pareja, lo que nos acerca a una gestión del cuidado, en estos pacientes, más integral e integradora, considerándolo un ser biopsicosocial en relación constante con el entorno.
\end{abstract}

Palabras clave: Neoplasia testicular, adulto joven, sexualidad.

\begin{abstract}
Cancer is a disease that causes social and human phenomenon very important, so the testicular cancer is the most common malignancy in men aged 15-35 years, one of the most curable solid neoplasms. This qualitative, phenomenological approach to describe the meaning they assign to their sexuality six young adults treated with chemotherapy for testicular cancer in the city of Puerto Montt, from March to December 2010, on their selfesteem, relationship partner and sexual life. Data analysis was performed by using the computer program Atlas Ti. It was noted that the significance the young adult gives to sexuality is understood by the self assessment and its environment, depending on the network of support that each one has, where the sexual life remains important, but not transcendent or primary. The couple is a key contributing factor to the formation of a life plan and is in that relationship that sexual life becomes relevant. Therefore, the physical effects caused by treatment, despite the inevitable implications involved, do not influence the self-image, sex life or relationship, which brings us to a careful management of these patients in a more comprehensive and inclusive way, regarding a biopsychosocial being in constant contact with the environment.
\end{abstract}

Key words: Testicular neoplasm, young adult, sexuality.

Fecha recepción: 26/04/11 Fecha aceptación: 14/04/12

\footnotetext{
* Enfermera, profesora Departamento de Enfermería, Facultad de la Salud, Universidad de Antofagasta, Chile. E-mail: carmenluz.munoz@uantof.cl, carmenluzmz@hotmail.com

** Enfermera, profesora Departamento de Pediatría y Cirugía Infantil, Facultad de Medicina, Universidad de La Frontera. Temuco, Chile.E-mail: xosorio@ufro.cl
} 


\section{INTRODUCCIÓN}

El cáncer es una enfermedad que provoca un fenómeno social y humano muy importante, más aún cuando afecta a personas productivas, en edad reproductiva y sexualmente activas, como es el adulto joven que se ve enfrentado al diagnóstico de cáncer testicular y a los aspectos relacionados con su enfermedad (1). El cáncer testicular es una enfermedad en la cual se encuentran células neoplásicas en uno o ambos testículos (2); es un cáncer del adulto joven, con una media de edad de 30 años (16-69 años) (3). Dada la importancia económica y moral que tiene para la sociedad una persona con esta patología, la/ el enfermera/o debe tomar un rol integrador junto a su familia y el sistema de salud, recogiendo fundamentos científicos que contribuyan con la disciplina en pos del usuario; con sensibilidad y creatividad para enfrentar los significados de la realidad multisensorial que incluye las necesidades biológicas, comunicativas, afectivas, sociales y culturales (4-6); por lo que, estando en conocimiento de cuáles son sus dudas, temores y preocupaciones, en el plano de la sexualidad, a la/ el enfermera/o le es posible fomentar con mayor éxito los pilares de la resiliencia en él, pareja y/o su familia.

La sexualidad hace referencia a un campo más extenso que el acto sexual, también incluye una amplia gama de actividades como las caricias, determinados olores, miradas, tonos de voz y expresiones (7-9). Teniendo en cuenta la experiencia de trabajar con personas en busca de su autodefinición (10), con una familia y/o pareja, que también debe asumir los cambios que conlleva el tratamiento y la enfermedad (7, 11-14), se planteó comprender ¿cuál es el significado que otorgan a su sexualidad los adultos jóvenes sometidos a tratamiento con quimioterapia por cáncer testicular, de marzo a diciembre del año 2010, en relación con los aspectos de su autoestima, relación de pareja y vida sexual?
La evolución de la enfermería ha permitido que la persona hospitalizada sea tratadas como un todo, en este sentido reconocer lo que significa someterse a un tratamiento como la quimioterapia es parte de este todo; se extreman los cuidados, deben dejar de trabajar o estudiar sin tener posibilidad de realizar actividades que pongan en riesgo su existencia; aparece una vida definida por reglas y normas que deben cumplir en el hospital, la casa y el entorno, debido a las complicaciones que provoca la inmunosupresión (1). Por otra parte, en hombres jóvenes tratados por cáncer testicular se produce una disminución de los niveles de testosterona, lo que puede provocar disminución del deseo sexual (3), además de poder verse dañado los reflejos de erección por los tratamientos (1), manteniéndose presente el deseo de proximidad y contacto físico (5). Según Roy, frente a lo anterior, debe existir una adaptación a estos cambios, donde la persona busca continuamente estímulos o procesos de afrontamiento que al final dan paso positiva o negativamente a esta adaptación (15); el conocimiento y la explicación de la realidad, en relación al medio que los rodea, puede predecir el comportamiento de esa realidad (16).

La sexualidad en adultos jóvenes con cáncer testicular $(2,3)$ ha sido poco estudiada, al ser un tema íntimo no abordado por los equipos de salud que trabajan atendiendo a este tipo de pacientes. En la literatura se encuentran estudios desarrollados en mujeres con cáncer de mama, homólogo al cáncer testicular en varones; uno de ellos, en donde participaron en un programa de intervención psicosocial, reveló que aquellas mujeres que estuvieron sometidas a una intervención muestran mejores resultados en lo relacionado a la imagen corporal. En cuanto al tipo de cirugía, las mujeres tumorectomizadas tuvieron una mejor imagen corporal que las mastectomizadas (17); así la autoestima, como uno de los ámbitos de la sexualidad, se encuentra más fácilmente en la literatura; 
para Alonso et al. (18), en su estudio descriptivo transversal sobre autoestima y relaciones interpersonales en jóvenes estudiantes, los resultados apuntalan los supuestos de la necesidad de apoyo frente a situaciones de conflicto. El estudio mostró que algunos estudiantes tienen una adecuada autoestima y son capaces de solucionar conflictos, sin embargo se encontró un número de adolescentes que adolecían de estas capacidades; Almaza et al. (19) subrayaron particularmente que el cuidado psicosocial del paciente con cáncer y su familia debe necesariamente ubicarse dentro del contexto social y cultural propio. El conocimiento y consideración de la dinámica familiar, social y global son requisitos esenciales para definir prioridades específicas.

En otro estudio cuyo objetivo era determinar la fiabilidad y la validez de la autoestima, en una muestra de adultos chilenos, mostró que ésta se relaciona directamente con el bienestar de las personas y podría ser un buen indicador de salud mental (20).

Por otra parte, la sexualidad ha sido estudiada en diferentes situaciones de padecimiento considerando al entorno y familia como un pilar fundamental para enfrentar dichas situaciones. En un estudio exploratorio con enfoque cualitativo, que trata del proceso de adolescentes con VIH/SIDA, ellos y sus familiares cuidadores manifestaron preocupación con los cambios propios de la época, especialmente en asuntos relacionados con la sexualidad, además del diagnóstico y la convivencia con el VIH con el fin de protegerlos (21).

La sexualidad, desde el punto de vista de las relaciones, ha sido un tema de interés también. Uno de los problemas que enfrentan los pacientes crónicos y discapacitados es la negación de su sexualidad por la sociedad y la familia (22); en un estudio, veinticinco adolescentes discapacitados y sus madres fueron evaluados con un test, previamente probado con adolescentes sin discapacidad. Éste mostró que la autoestima (test de Coo- persmith) y los conocimientos adquiridos en reproducción y afectividad aumentaron significativamente y se mantuvieron a 6 meses de seguimiento. Los conocimientos de las madres también presentaron un aumento significativo. Reconocer los sentimientos de las madres podría mejorar la relación médico-paciente-familia. Esta metodología de trabajo se recomienda para la educación en afectividad y sexualidad, así como para el apoyo integral a este tipo de adolescentes y sus familias (22).

El presente estudio pretende describir el significado que otorgan a su sexualidad los adultos jóvenes sometidos a quimioterapia por neoplasia testicular en Puerto Montt, desde marzo a diciembre del 2010, a partir de la opinión de los propios sujetos indagando sobre los significados otorgados en relación a su vida sexual; develando su relación de pareja durante el proceso de quimioterapia y explorando en la percepción de su autoimagen durante el tratamiento; planteándose como supuestos que estos adultos jóvenes verían alterada su vida sexual debido a los cambios físicos y la alteración de la imagen corporal que producen las drogas; sus relaciones de pareja se deterioran por el impacto del diagnóstico, los cambios físicos y los cambios que deben realizar en su forma de vida debido a la inmunosupresión y que su autoimagen y el concepto de sí mismos se perturba al enfrentarse a los efectos secundarios del tratamiento, lo que afectaría también su relación con el entorno.

\section{MATERIAL Y MÉTODO}

La investigación se fundamentó en una metodología cualitativa-descriptiva con un enfoque fenomenológico, indagando en los relatos que hacen acerca de la realidad y la experiencia de vida de los adultos jóvenes sometidos a quimioterapia por cáncer testicular y los significados que se relacionan con lo 
que ellos narran sobre su sexualidad, autoestima, relación de pareja y vida sexual (23-27), donde juega un papel importante su entorno familiar, social y humano (28). Los sujetos de estudio son adultos jóvenes, con diagnóstico de neoplasia testicular, que fueron sometidos a tratamiento con quimioterapia, correspondiendo a seis adultos jóvenes de entre 20 y 40 años; con pareja heterosexual estable; sin enfermedad invalidante concomitante; con primer diagnóstico de cáncer y que sepan leer y escribir, como criterios de inclusión.

Para la recolección de los datos se utilizó una entrevista semiestructurada, que permitió sondear razones, motivos y ayudar a comprender el significado de la sexualidad (29, 30). Las entrevistas fueron realizadas por las investigadoras durante los meses de octubre a diciembre del año 2010, utilizando grabaciones magnetofónicas, para registro fiel de todas las interacciones verbales (27). Se solicitó firmar Consentimiento informado.

El estudio consideró los requisitos éticos propuestos por Ezequiel Emanuel (31) y se contó con la aprobación del Comité de Ética del Hospital de Puerto Montt. Se consideraron, además, los criterios de rigor propuestos por Guba y Lincoln $(32,33)$, como son la credibilidad, transferibilidad, consistencia y neutralidad, que dan fe de la rigurosidad y veracidad de los resultados y su análisis.

El tratamiento de los datos que se realizó fue por categorías: autoimagen, relación de pareja y vida sexual, donde, una vez transcritas las entrevistas, fueron organizadas, preparadas, analizadas e interpretadas con el programa computacional Atlas Ti. La evaluación de los resultados fue realizada por el investigador con apoyo de un experto en métodos cualitativos, quien hizo revisión del análisis, emitió opinión y entregó sugerencias a cada uno de los pasos comprendidos en el proceso, sin ser un integrante activo de la investigación (28).

Para efectos del análisis "autoimagen" se entiende como "concepto de sí mismo" o "autoconocimiento", entrando aquí toda cla- se de actividades y contenidos cognitivos, la autoimagen es la forma en la que cada ser se valora y por ende confía en sí mismo $(11,12)$, íntimamente ligada al concepto de sexualidad está la "relación de pareja" que hace referencia a una unión dependiente entre sí, por lo que afecta al deseo y a la capacidad de dar a los demás y de recibir de ellos aspectos de todo lo que uno puede ofrecer, como amor, respeto, valores, educación, conocimientos, habilidad, responsabilidades, bienes materiales, tiempo y talento (13). Por último, la "vida sexual" se define como un estado dentro de la sexualidad que sigue siendo una actividad vital de la persona que cursa una enfermedad, aún en estadios avanzados y aunque disminuya el interés por el coito, se mantiene presente el deseo de la proximidad y el contacto físico (3).

\section{RESULTADOS}

De acuerdo al primer objetivo: "Indagar sobre los significados otorgados por los adultos jóvenes en relación a su vida sexual", donde la categoría es "vida sexual", se observa en función del discurso planteado la importancia de ésta para los sujetos de estudio. Se visualiza la presencia de tres subcategorías: "Tenencia de vida sexual" (existencia de vida sexual activa), "Importancia de la vida sexual" (valor que le otorga el adulto joven a la presencia de una sexualidad activa, aludiendo a la sensación de virilidad masculina) e "Implicancias en la vida sexual" (consecuencias o efectos que ha generado la aplicación del tratamiento en el ámbito sexual) y se observa la relación existente entre ellas; así, al considerar tenencia de vida sexual, presente en el 100\% de los sujetos, se manifiesta que está asociada, pero no en su totalidad, a la importancia de ésta, ya que para algunos la vida sexual siempre fue importante, para otros dejó de serlo luego del tratamiento, por tanto, independiente de la presencia de una 
vida sexual activa, la importancia se visualiza, más bien, como un tema de masculinidad. Por otra parte, no ocurre lo mismo con las implicancias en la vida sexual, donde a pesar que en algunos casos el tratamiento sí generó dificultades físicas que repercutieron en aspectos como el deseo sexual o malestares físicos, esto no ha sido un impedimento para el logro de una vida sexual activa con normalidad.

En relación al segundo objetivo: "Develar la relación de pareja de los adultos jóvenes durante el proceso de quimioterapia", siendo "relación de pareja" la categoría establecida, se desprende la presencia de cinco subcategorías asociadas: "Proyecto de vida" (expectativas futuras en torno a la formación de una vida estable con la pareja), "Redes de apoyo" (círculo de personas, ya sean familiares, amigos, pareja, entre otros, quienes estuvieron presentes y fueron un soporte importante en el proceso del tratamiento de la enfermedad), "Cambios negativos en la relación de pareja" (cambios que perjudicaron la relación de pareja producto de los efectos, tanto físicos como sicológicos, que generó el tratamiento), "Estabilidad en la relación de pareja” (mantención de la comunicación y en el trato de la relación de pareja en el proceso del tratamiento) y "Cambios positivos en la relación de pareja" (aspectos de mejora en la relación luego del tratamiento). Se observa que las redes de apoyo, los cambios negativos y positivos en la relación de pareja y la estabilidad, son cada una por sí sola parte de la subcategoría "proyecto de vida". Por otra parte, las redes de apoyo están asociadas a los cambios negativos, positivos y estabilidad en la pareja; esto, porque los cambios generados, o la estabilidad que se pudo haber creado en el proceso, además del apoyo recibido por la pareja, fueron elementos fundamentales al momento de visualizar sus prioridades futuras en función de aspectos como familia, trabajo, adquisición de bienes, entre otros, la intensidad y la forma en cómo ha recibido apoyo, ha afectado en la trascendencia y el significado de su relación, lo que en algunos casos ocasionó cambios positivos, en otros negativos y en otros se mantuvo estable.

En síntesis, se puede visualizar que el proyecto de vida y las redes de apoyo son aquellas subcategorías con mayor influencia en la relación de pareja, donde los cambios positivos o negativos y la estabilidad de ésta están asociados a las influencias o quiebres en el proyecto de vida que tiene el adulto joven después del tratamiento, así como también estos cambios se generan producto del apoyo recibido de parte de su pareja.

De acuerdo a lo expuesto en el tercer objetivo: "Explorar la percepción del adulto joven en relación a su autoimagen durante el tratamiento", donde la categoría es "autoimagen", se observa la presencia de cuatro subcategorías que explican la incidencia del tratamiento en este ámbito: "Efectos físicos del tratamiento" (secuelas que ocasionó el tratamiento en el aspecto físico), "Redes de apoyo" (círculo de personas, ya sean familiares, amigos, pareja, entre otros, quienes estuvieron presentes y fueron un soporte importante en el proceso del tratamiento), "Reacción a la enfermedad" (forma como sobrellevó la enfermedad en el primer impacto de la noticia y en el proceso propiamente tal, tanto a nivel personal como familiar) y las "Consecuencias en la autoestima" (efectos que inciden en la valoración personal del adulto joven producto de la enfermedad). Se observa que los efectos físicos del tratamiento ocurridos por la quimioterapia son parte de las consecuencias en la autoestima del adulto joven, donde sucesos como la caída del pelo y los dolores generados por el tratamiento hacen que el individuo se sienta con menos energías o decaído, lo que repercute en su estado anímico. Por otra parte, las redes de apoyo también son parte de las consecuencias en la autoestima, ya que en términos generales refieren que el apoyo de la familia y pareja son fundamentales para superar con mayor normalidad las secuelas de la enfermedad. De otro modo, la reacción frente a 
la enfermedad se visualiza más cercana con las consecuencias en la autoestima, por tanto se observa no sólo como parte sino como causa de ésta; esto porque la noticia de la enfermedad inevitablemente incide en la valoración propia. Además, la reacción frente a la enfermedad se asocia a los efectos físicos del tratamiento ya que en el proceso de éste, dependiendo de los efectos que produjo en el adulto joven, éste fue cambiando su reacción frente a la enfermedad, donde en algunos casos mejoró y en otros decayó. Por último, la reacción frente a la enfermedad también se asocia a las redes de apoyo, esto porque el soporte de la familia, pareja y amigos interfirió en la percepción de la enfermedad que fue manifestando el adulto joven en el proceso, por tanto también implicó un cambio en la reacción frente a ésta.

En síntesis, el concepto de autoimagen está asociado a la presencia de los efectos tanto físicos como psicológicos; de esta forma, la valoración positiva del adulto joven va a depender de cómo se manifiestan estos aspectos. Así, se observa que los elementos asociados a la reacción de la enfermedad, como son las redes de apoyo y efectos físicos del tratamiento, son causa determinante en las consecuencias tanto positivas como negativas en la autoestima del adulto joven en cuestión.

\section{DISCUSIÓN Y CONCLUSIÓN}

Considerando la importancia de la sexualidad para la vida de las personas en general y más aún para el tramo etario que corresponde al "adulto joven", se observa que a pesar de la magnitud que conlleva una enfermedad como el cáncer testicular, que afecta aspectos de índole físicos y psicológicos, se puede identificar una serie de elementos que contribuyen a la disminución de las secuelas. Así, el concepto de sexualidad se explica en esta investigación, a través de la unión y conexión de tres elementos relevantes como lo son: autoimagen, relación de pareja y vida sexual.

En primera instancia se plantea que la autoestima está íntimamente ligada al concepto de sexualidad incidiendo en sentimientos, el grado de competencia, aceptación y proximidad con otras personas (12). Así, se observa que en los resultados obtenidos existen aspectos como la reacción a la enfermedad, redes de apoyo y efectos físicos de la enfermedad que han interferido en la autoestima. En relación a la percepción del adulto joven en cuanto a su autoimagen, en algunos casos el hecho de conocer el padecimiento de esta enfermedad de manera fortuita generó gran conmoción y sentimientos de decaimiento, miedo y preocupación tanto por las repercusiones físicas y de salud, así como también por sus familias y pareja. No obstante, a medida que se fue desarrollando el tratamiento, a pesar de los efectos que produjo, se dieron cambios en la forma cómo sobrellevar la enfermedad. Si lo llevamos al concepto de Roy (15), se podría inferir que independiente de las consecuencias a corto plazo que ocasionó el tratamiento y la información de la existencia de ésta, el apoyo recibido tanto por su entorno cercano como del equipo de salud, permitió que su concepto de autoimagen no se sintiera menoscabado, lo que ayudó al adulto joven a valorar la importancia de ellos frente a su entorno. Es así como al adulto joven, si bien le fue difícil enfrentar las secuelas de tal enfermedad, por los efectos de ésta, pudo sobrellevar tal experiencia de la mejor forma a su alcance. En lo relativo a la autoimagen los principales elementos implicados están asociados en mayor medida con aspectos de relaciones de apoyo, más que con efectos corporales o físicos del tratamiento. Así, la/el enfermera/o cumple un rol importante a lo largo de todo el tratamiento, brindado apoyo psicológico cuando enfrenta el diagnóstico, contención en el proceso que involucra la enfermedad y su tratamiento, a través de la atención y educación, y frente a 
la radioterapia o quimioterapia debe brindar cuidados en cada una de las situaciones que enfrenta el joven junto a su familia, sus redes de apoyo y el equipo de salud.

Los efectos físicos que el tratamiento ocasionó en ellos, a pesar de los cambios en la imagen corporal y las implicancias físicas de la enfermedad, tanto a nivel sexual como funcional, no se manifiestan como relevantes y sustantivas en los cambios y en el proyecto de vida; así en las relaciones de pareja se plantea que el poder de los hombres está apoyado en la legitimidad general que tiene la dominación masculina en la sociedad y en predominio de sus necesidades sexuales, donde también factores de orden psicológico, como las creencias, impacto emocional que provoca una enfermedad, motivaciones y afrontamientos, tienen gran importancia en la aparición de dificultades del orden sexual $(5,8)$. No obstante, al observar los aspectos relacionados con esta categoría, se puede visualizar que la estabilidad en la relación de pareja y los cambios, tanto positivos como negativos, se han generado a partir de una relación social entre los dos individuos, más allá de la relación sexual y de manera tal que los factores de índole psicológico y emocional priman sobre la dominación masculina en cuanto a las necesidades sexuales que se mencionan anteriormente. Esto se ratifica a partir de la construcción del proyecto de vida, en donde se ve menoscabado producto de los cambios negativos que se generaron en la relación de pareja luego del tratamiento, o bien, se reafirma un proyecto de vida en pareja producto de aquellos cambios positivos.

En relación a la vida sexual se plantea que personas con cáncer testicular experimentan situaciones que conllevan una disminución de los niveles de testosterona, lo que puede provocar una disminución del deseo sexual $(5,8)$. Los tratamientos contra el cáncer, desafortunadamente, pueden dañar los reflejos de erección a través de una serie de mecanismos. Las causas hormonales que intervienen en la disminución del deseo sexual ha- cen que presenten dificultades para lograrla, sin embargo algunos hombres, sobre todo los menores de 50 años, pueden lograr erecciones normales aun con niveles insuficientes de testosterona. No obstante, se observa que a pesar de tal situación, en los resultados obtenidos no se manifiesta una pérdida de la actividad sexual, ya que se identificó que independiente de la importancia y las implicancias que manifiesta el adulto joven en relación a su vida sexual, esto no repercute en la tenencia de ésta. Quedó de manifiesto que estos factores han variado de acuerdo a cada caso y a los efectos físicos después del tratamiento; es así como se deduce que la vida sexual tiene una connotación ligada a la interacción y relaciones interpersonales, así como también a aspectos de índole psicológico y en menor medida fisiológicos, donde la autoestima cumple un rol primordial. Se identifica a la subcategoría "redes de apoyo" como una de las que tiene mayor influencia y por tanto con mayor asociación con otras subcategorías, explicando de cierta forma la relación entre el concepto de autoimagen y relación de pareja. Roy plantea sobre el concepto de las relaciones (15), que la unión y los lazos que posee el individuo han afectado en el deseo y capacidad de dar a los demás y de recibir de ellos aspectos de todo lo que uno puede ofrecer, tales como: amor, respeto, valores, educación, conocimientos, habilidad, responsabilidades, bienes materiales, tiempo y talento. Lo anterior, va más allá de una relación de pareja, sino más bien de relaciones familiares y de amistad, donde el individuo debería ser capaz de entregar todos estos aspectos, aun en momentos de sufrimiento o padecimiento. Se puede deducir que la forma cómo se manifiestan las redes de apoyo, las que se observaron predominantemente por parte de familiares, en especial la madre, y en gran importancia la pareja, podría repercutir de manera directa en la autoestima del individuo que padece esta enfermedad. En virtud de lo anterior, y aludiendo nuevamente al concepto de autoestima de Roy, identifica 
la relación de la subcategoría "redes de apoyo" con "proyecto de vida", "reacción a la enfermedad" y las subcategorías asociadas a los cambios y estabilidad en la relación de pareja, donde el individuo actúa y se desenvuelve en función de su enfermedad de acuerdo a su relación con el grupo, pareja, familia y amigos, cada uno por sí solo y en su conjunto.

Callista Roy en su modelo se centró en la adaptación que la persona logra frente a las situaciones de cambio (15); como se observó en la presente investigación, el diagnóstico y tratamiento del cáncer de testículo produjo una serie de cambios importantes en la persona y su familia, éstos dependen también de sus sistemas de apoyo, del estilo empleado por ellos mismos para afrontarlos o de su historia; el objetivo del modelo de Roy es que el individuo, como un ser biopsicosocial, llegue a un máximo nivel de adaptación y evolución, en lo concerniente a la fisiología, autoimagen, el dominio del rol y la interdependencia con ese entorno que también se modifica. Es así como se observa una íntima conexión entre la autoimagen, relación de pareja y vida sexual, ya que cada una de las variables que involucran estos conceptos están asociados directa o indirectamente, $y$ con distintos grados de intensidad, lo que deja en evidencia que la repercusión de esta enfermedad no sólo alude a aspectos corporales, sino más bien a aspectos emocionales o de relaciones de apoyo, convirtiéndose en un soporte para solventar de mejor forma las repercusiones, cambios físicos y psicosociales que afecta el tratamiento y la enfermedad en sí. De acuerdo a esto, el desafío es determinar una serie de acciones, como procesos de afrontamiento que expone Roy, que contribuyan a equilibrar estos aspectos para aminorar los cambios negativos que pudieran presentarse tras la aparición del cáncer testicular en la población adulto joven.

Por último, como primer acercamiento investigativo, y como una forma de contribuir al conocimiento y gestión del cuidado de pacientes que padecen cáncer testicular, se consideraron aquellos aspectos negativos que fueron presentando en el discurso y que al rescatarlos sirven como base para la creación de líneas y alternativas de acción para la prevención y tratamiento, con el fin de mejorar los procesos y por ende la calidad de vida durante y postratamiento; no existe evidencia científica suficiente que permita conocer cuál es el mejor método de detección precoz del cáncer de testículo, aunque se sugiere que el autoexamen testicular y el screening mediante ecografía testicular son métodos efectivos para una detección precoz de este cáncer (34); por ello, el aspecto de promoción y prevención en salud es importante; es decir, promover en los jóvenes una actitud positiva y responsable en relación a su sexualidad, instruyendo a la población en aspectos de la educación sexual integral e incluyendo técnicas o métodos de autoexamen testicular, donde la/el enfermera/o, dados sus conocimientos, podría liderar el equipo de salud en este abordaje.

\section{REFERENCIAS}

1. Arraztoa J. Cáncer. Diagnóstico y tratamiento. Madrid: Mediterráneo; 1998.

2. Ministerio de Salud de Chile. Guías Clínicas Cáncer de Testículo en personas de 15 años y más [Internet]. Santiago, Chile: Ministerio de Salud; 2005. [citado 04 noviembre 2009]. Disponible en: http:// www.minsal.cl

3. Departamento de estadísticas e información en salud [Internet]. Santiago, Chile: Ministerio de Salud; 2005 [citado 15 diciembre 2009]. Disponible en: http:// deis.minsal.cl/index.asp

4. Long B, Phipps W, Cassmeyer V. Enfermería médico quirúrgica. Un enfoque del proceso de enfermería. $3^{a}$ edición. Madrid: Harcourt Brace; 1998.

5. Grau J, Llantá M, Chacón M, Fleites, G. La sexualidad en pacientes con cáncer: 
algunas consideraciones sobre su evaluación y tratamiento. Revista cubana de Oncología 1999; 15(1): 49-65.

6. Diccionario de la lengua española. $2^{\mathrm{a}}$ ed. [Internet]. 1992 [actualizada el 2001; citado 30 diciembre 2009]. Disponible en: $\mathrm{http} / / /$ lema.rae.es/drae/?val=significado

7. Masters W, Johnsons V, Kolodny R. La sexualidad Humana. 10 a edición. España: Grijalbo; 1997.

8. Botella J. Sexualidad en el climaterio, en la edad crítica. $9^{a}$ edición. Barcelona: Salvat; 1990.

9. Suárez EA, Cortina MI. La familia. Rev Sexol Soc. 1996; 2(5): 6.

10. Luengo M, Toledo V, Fuentes M, Lobos $\mathrm{L}$, Molina R et al. Adolescentes discapacitados: talleres de afectividad y sexualidad. Rev Chil Pediatr. 2000; 71(1): 24-31.

11. Valdés T, Benavente M, Gysling J. El poder en la pareja, la sexualidad y la reproducción. Mujeres de Santiago. [Internet]. Santiago, Chile: Serie Libros FLACSO; 1999 [citado 02 agosto 2010] 178p. Disponible en: http://cronopio. flacso.cl

12. Cataño D, Restrepo S, Portilla N, Ramírez H. Autoestima y sexualidad en adolescentes: validación de una escala. Investig. andina. 2008, 10(16): 57-66.

13. Manrique S. El amor y la consulta: Relaciones amorosas y problemas médicos. Medifam. 2002; 12(10): 641-45.

14. Johansen O. Introducción a la teoría general de sistemas. [Internet] México: Limusa; 2008 [citado 02 agosto 2010]; [178p.]. Disponible en: http://www. worldcat.org

15. Marriner T, Raile A. Modelos y Teorías en Enfermería. $5^{a}$ edición. España: Elsevier; 2003.

16. Johansen O. Introducción a la teoría general de sistemas. $12^{\mathrm{a}}$ edición. México: Limusa; 1996.

17. Sebastián J, Manos D, Bueno M, Mateos N. Imagen corporal y autoestima en mujeres con Cáncer de mama participantes en un programa de intervención psicosocial. Clínica y salud. 2007; 18(2): 13761.

18. Alonso L, Murcia G, Murcia J, Herrera D, Gómez D, et al. Autoestima y relaciones interpersonales en jóvenes estudiantes de primer semestre de la división salud de la Universidad del Norte, Barranquilla (Colombia). Salud Uninorte. 2007; 23(1): 32-42.

19. Almaza J, Holland J. Psico-oncología: estado actual y perspectivas futuras. Rev Inst Nac Cancerol. (Mex). 2000; 46(3): 196-206.

20. Rojas-Barahona C, Zegers B, Förster C. La escala de autoestima de Rosenberg: Validación para Chile en una muestra de jóvenes adultos, adultos y adultos mayores. Rev Med Chil. 2009; 137(6): 791800.

21. Lima A, Pedro E. Creciendo con VIH/ SIDA: un estudio con adolescentes portadoras de VIH/SIDA y sus cuidadoras familiares. Rev Lat Am Enfermagem. 2009; 16(3): 348-54.

22. Ríos A. Alcance de las políticas públicas en el área de salud sexual y reproductiva dirigidas a los adolescentes en Colombia. Salud Uninorte. 2008; 24(2): 351-58.

23. Rodríguez G, Gil J, García E. Metodología de la investigación cualitativa. $2^{\mathrm{a}}$ edición. Granada: Aljibe; 1999.

24. Ruiz J. Metodología de la investigación cualitativa. Bilbao: Universidad de Deusto; 1996.

25. Hernández R. Metodología de la investigación. $4^{\text {a }}$ edición. México: Mc GrawHill; 2006.

26. Bello A. Fenomenologia e ciências humanas: psicologia, história e religião. Capitulo: Pressupostos Históricos. [Internet]. Bauru, SP: EDUSC; 2004 [citado 27 diciembre 2009]. Disponible en: http://www.fafich.ufmg.br

27. Leal Riquelme R. Aportes teórico-metodológicos de la fenomenología al desarrollo cualitativo de las ciencias sociales 
en Alfred Schütz. Alpha. [Internet] 2007; 25. [citado 29 diciembre 2009]. Disponible en: http://www.scielo.cl

28. Amezcua M, Gálvez A. Los modos de análisis en investigación cualitativa en salud: perspectiva crítica y reflexiones en voz alta. Rev Esp Salud Publica. 2002; 76(5): 423-36.

29. Valles A. Técnicas cualitativas de investigación social. Madrid: Síntesis; 1999.

30. Valles A. Técnicas cualitativas de investigación social. Reflección metodológica y práctica profesional. $4^{\mathrm{a}}$ reimp. Madrid: Síntesis; 2007.

31. Rodríguez E. Comités de evaluación ética y científica para la investigación en seres humanos y las pautas CIOMS 2002. Acta Bioeth [Internet]. 2004 [citado 22 agosto 2010]; 10(1): 37-48. Disponible en: http://www.scielo.cl

32. Guba E, Lincoln Y. Naturalis Inquirí. London: Sage; 1985.

33. Vázquez L, Delgado $M$, Fernández $M$, Mogollón A, Ferreira M, Vásquez M. Introducción a las técnicas cualitativas de investigación aplicadas en salud. España: Universidad Autónoma de Barcelona; 2006.

34. Cambil, J. Efectividad del autoexamen y del screening en la detección de Cáncer de testículo. Index Enferm. 2005; 14(1): 48-9. 